\title{
Social and economic factors in prosthodontic practice and education
}

\author{
Lisa A. Tedesco, PhD, a and Davis A. Garlapo, DDs \\ University of Michigan, School of Dentistry, Ann Arbor, Mich., and School of Dental Medicine, \\ State University of New York at Buffalo, Buffalo, N. Y.
}

\begin{abstract}
Prosthodontics represents a highly developed body of knowledge and skill that spans multiple disciplines. Numerous studies can be cited that address biologic, mechanical, or materials science factors that influence decisions about patient care. On a daily basis as clinicians, teachers, or patients, we experience an interplay of social, economic, and psychologic conditions that similarly influence treatment decisions. This article explored a rationale for including a clear, explicit emphasis on knowledge and skill development for prosthodontic practice and education related to social, psychologic, and economic factors. (J Prosthet Dent 1994;71:3105.)
\end{abstract}

Multiple forces are at work shaping the actions dentists take on behalf of their patients. Some actions are patient-initiated, and some are not. As care providers or as patients there is an interaction of social, economic, and psychologic conditions that influence clinical judgment and treatment. Explicit and implied social and economic factors that influence prosthodontic practice are identified. Information from the social sciences is reviewed for sharpening the patient focus in prosthodontic care and education.

\section{Sharpening focus on the patient: Why now?}

Social and economic factors that influence prosthodontic treatment provide an interesting challenge. Science, technology, and oral epidemiology continue to broaden the scope of prosthodontic treatment. Social and economic factors also have the potential to influence the broad scope of prosthodontic treatment. Over the past 10 years, there has been an abundance of articles that describe and evaluate the limits placed on patient treatment relative to treatment planning, occlusal and anatomic evaluation, biomaterials, biomechanical aspects of treatment, prosthodontic fabrication, and serviceability. ${ }^{1-3}$ The published "Principles, Concepts, and Practices in Prosthodontics"2 mentions gathering diagnostic information to include the patient's concerns and expectations, with this information noted in the patient record. Recommendations are made with respect to patient education, but ggain the focus is on

Presented at the Academy of Prosthodontics meeting, Vancouver, B.C., Canada.

aProfessor and Associate Dean for Academic Affairs, University of Michigan, School of Dentistry.

bProfessor and Chair, Department of Fixed Prosthodontics, School of Dental Medicine, SUNY at Buffalo.

Copyright $\odot 1994$ by The Editorial Council of THE JOURNAL OF

Prosthetic Dentistry.

$0022-3913 / 94 / \$ 3.00+0 . \quad \mathbf{1 0 / 1 / 5 2 2 6 6}$ the limits of treatment. There is no mention of comprehensive, individualized social or psychologic evaluation of the patient and related protocol design that will maximize success in prosthodontic care.

Implant dentistry has dramatically increased the range of oral rehabilitation possibilities for patients. At the same time, implant dentistry is by definition invasive and complex. Increasingly, the literature in prosthodontics, periodontics, and oral and maxillofacial surgery demonstrates the influence of patient factors such as cooperation and satisfaction in treatment success. ${ }^{4-6}$ Increasingly, research and clinical experience indicate that comprehensive evaluation of the patient and a protocol sensitive to the patient's individual characteristics are needed for treatment success. Whereas dentists have always been interested and sensitive to the social, psychologic, and economic characteristics of their patients, the increasing use of dental implants requires refocusing on patient characteristics and patient satisfaction.

\section{Patient arts and protocol design}

One of the best and earliest examples of comprehensive, systematic evaluation of social, psychologic, and economic characteristics of the patient seeking prosthodontic care can be found in the text of Brewer ${ }^{7}$ in Mastering the Art of Complete Dentures. Brewer's clinical approach emphasized general appraisal of the patient, which included dress, carriage, poise, the handshake, aging and aging prognosis; patient needs, expectations, and attitudes; and medical, psychologic, and behavioral considerations. For Brewer, these appraisals were an integral part of diagnosis and treatment planning. Most of this information was gathered through simple observation, standard questioning, and careful listening. Except for the Cornell Medical Index, designed to assess psychologic and behavioral problems associated with medical conditions, no other standardized psychologic assessments by trained or authorized clinical 
psychologists were performed. According to Brewer and his colleagues, they were careful to follow the established protocol for interpreting the results from the Cornell Medical Index. In the case of complete denture treatment, this index has been found to predict patient dissatisfaction. ${ }^{8}$

The authors describe how the dentist-patient interaction should proceed to enhance patient satisfaction during the final stages of prosthodontic rehabilitation. For example, patient approval of the final restorations should not be rushed and it is suggested that the patient look at his or her teeth while standing in front of a large mirror, instead of sitting and holding a hand mirror. The intention is to have the patient place the restorations in the context of the whole face and upper body during the self-evaluation process. In this way, patients who are inclined to scrutinize details will be dissuaded from focusing on small areas of tooth surface and gingival margins. Also recommended for patients who do not open their mouths to look or just briefly check their restorations, extra time should be given to ask about their satisfaction.

Health psychologists and social scientists currently working to improve dental implant treatment success use the conceptual framework, which places the patient, along with social and psychologic characteristics, as the center for treatment decision-making.

\section{Lessons from implant dentistry}

At the center of approaches offered by clinical and research psychologists working in the dental implant area are recommendations for understanding all of the tasks each patient and practitioner faces. ${ }^{4,9}$ Patient-based research is growing; however, most studies are done on dental school or hospital-based clinic samples with highly trained interdisciplinary teams of specialists in clinical dental and psychological sciences. In these facilities, treatment success is more likely than not simply because of experience and expertise. Deciding which patients are at risk for treatment dissatisfaction, which patients to interview or "assess" extensively, is still a difficult decision because there is still much to learn about the psychologic and social aspects of treatment success or failure.

Dental research, clinical practice, and behavioral medicine offer some guidelines that can help the treatment process for the patient and clinician. On the patient's side, for example, important variables include expected outcomes from the rehabilitation beyond comfort and function; expected cooperation and tolerance for the rehabilitation process that includes pain and discomfort; capacity to comply with a maintenance regimen, including assessment of variables that interfere with compliance, such as busy and multiple demands for time, and depression or anxiety disorders that interfere with sustaining a routine. The guidelines recommended on the dental care provider side are (1) available time and personal capacity to observe, describe, and derive meaning from the social and psychologic characteristics of each patient; (2) consulting relationships with mental health professionals; (3) ability to provide informed consent and verify patient understanding of treatment risks; and (4) provision of follow-up care for psychologic and emotional acceptance of the treatment process.

The summary of guidelines suggests that the psychologic and social aspects of patients and care providers that characterize successful treatment with dental implants are similar to the characteristics that are associated with a successful denture. ${ }^{7,10,11}$

\section{Demography: age, wealth, and treatment seeking}

The average age and income for North Americans are increasing but are not uniform. In the United States, for example, there is a great deal of diversity of income and net worth in the aging population. ${ }^{12}$ Persons over 65 in the highest fifth for net worth are 90 times wealthier than those in the lowest fifth. In 1986, $\$ 859$ billion dollars represented the total income of households with at least one person over age 50, and $\$ 139$ billion of this total income figure was discretionary income. ${ }^{13}$ Although this economic diversity is certainly not restricted to the United States, it does have implications for patients seeking restorative, rehabilitative, and esthetic oral health care. Of all the dental services, diagnostic, preventive, surgical, or restorative, prosthodontic service with or without third party payment is most dependent on patients' ability to accept at least part of the cost of treatment, and, in countries where cultural values and health care financing arrangements include voluntary personal spending for oral health care costs, individuals will be faced with the most rapid and dramatic cost increases for prosthodontic care. In countries where oral health care is financed partially or totally by government resources, individuals may not sense the increases in cost immediately or directly. For example, indirect accommodations to absorb or defer costs may include limiting treatment options.

The change in oral epidemiology ${ }^{14-16}$ and disease prevention afforded by fluoride is perhaps the most successful preventive advance in all health areas. The post-World War II birth cohort did not have the childhood benefit of fluoride. Spanning approximately two and a half decades, this age group is a large demographic segment. The restoration and replacement of teeth represent treatment needs that are equally large. It is estimated that in the United States, the next 30 years will show an increase in fixed prosthodontic treatment need, based on the increased retention of posterior teeth. ${ }^{15}$ Some oral epidemiology analysts predict that the pattern of missing teeth could stimulate 106 million Americans to seek treatment that includes dental implants.

Projected trends for removable prosthodontic treatment appear to be diversely distributed in the older age groups and depend even more on financial resources available for dental care. The population over 65 years old will grow from 27 million to 64 million in the United States alone by the 
year 2020. By the year 2010 , nearly $49 \%$ of the United States population will be 40 years old or older, and between 1990 and 2010, the total population aged 50 years and older will show a $48 \%$ growth from 65 million to 97 million. By contrast, there will be a $1 \%$ population increase of persons under 50 years of age in this same 20 -year period.

\section{FROM "YUPPIE" TO “GRUMPIE"}

There are cultural media influences in addition to age, financial resources, and oral epidemiology as potential factors that influence patient desire for treatment or perceived need for treatment. In "free market" economies, demographers and marketers are special friends, particularly in the health care sector, where some health services are perceived as discretionary.

Last year, headlines in the business section of the New York Times read, "Shift for Marketers: Yup to Grump." The North American young urban professional, once called a "yuppie," is fast becoming the grown-up mature professional, or "grumpie." The newspaper article detailed the continuing courtship of post-World War II baby-boom generation consumers by marketers. ${ }^{17}$ In North America, marketers are stimulating the sale of consumer goods that ease this generation into facing the natural changes that come with aging. Most individuals experience these changes as decline, less stamina, less muscle tone, creaking knees, aching backs, weaker vision, facial creasing and wrinkles, periodontal disease, and the restoration of previously restored teeth. All forms of media are cleverly used to pursue market share. "Successful aging" is available for all who can afford to buy goods and services that promise to preserve structure, reduce deterioration, enhance comfort, and restore esthetic appeal. In the United States, this marketing courtship is aimed at approximately 78 million post-World War II baby boomers. Although this marketing strategy describes the North American scene particularly well, the response to aging and desire for related consumer goods of the post-World War II generation is a shared phenomenon in the industrialized world.

\section{THE ROLE OF FACIAL ESTHETICS AND PERCEPTION*}

Facial appearance, whether it is described from a psychological, social, or clinical perspective, remains a perceptual phenomenon. ${ }^{18}$ Whereas individuals within different cultures and societies embrace common definitions for facial and physical attractiveness and unattractiveness, how individuals respond to their own appearance is ultimately a personal and private matter shaped by the transactions of everyday life and is often independent of culture.

\footnotetext{
*Portions of this section were adapted from Albino JE, Alley TR, Tedesco LA, Tobiasen JA, Kiyak HA, Lawrence SD. Esthetic issues in behavioral dentistry. Ann Behav Med 1990;12:148-55, with permission.
}

Facial appearance provides a great deal of social and psychologic information. Age, gender, health, emotional state, individual identity, intelligence, and personality are typically discerned from facial appearance. Facial appearance can also influence how we perceive, think, and feel about each other. Expectations and impressions influenced by facial attractiveness are real, and affected are judgments of mental health, popularity, social class, intelligence, and aggressiveness. In social behavior studies of dating, physical attractiveness, not personality, interest, or scholastic aptitude is generally the best predictor of repeated dating of the same partner. Further, attractive men and women generally date more often and marry at earlier ages. Compliance with requests, desires, and demands are more likely to occur for attractive individuals than for unattractive individuals. Studies show that facial attractiveness increases the chances of receiving assistance and reduces blame and punishment in ordinary and criminal settings.

The physiognomic bases for making social judgments is the practice of judging character or psychologic qualities from facial features. While these judgments are seldom valid, oral characteristics rank high. Thin lips are judged to make people look dominant, sociable, or energetic. Missing or prominent incisors give the impression of aggressiveness; facial scars cause people to be judged dishonest; and people with prognathic profiles are viewed as more ambitious, determined, and dominant.

How facial appearance influences our feelings about ourselves is the other side of the perception coin. Social interaction provides a clear explanation for the development of self-perception of attractiveness. When individuals receive social feedback about themselves that is consistent with commonly held stereotypes, behavior often becomes consistent with the stereotype. Taken to its logical conclusion, individuals with facial impairments are likely to have more difficulty with social interactions than people without visible impairments and, consequently may have lower expectations and self-esteem for their own performance in any domain. This process is more commonly known as the "self-fulfilling prophecy."

Observers attribute more positive social characteristics to those with attractive facial appearance. Children with ideal dental facial appearance are perceived as more attractive, more desirable as friends, more intelligent, and less inclined to aggression that those with impaired dentalfacial appearance. Researchers have found that teeth represented the fourth most common target of teasing, after height, weight, and hair.

The psychologic and social issues related to esthetics in prosthodontic treatment may be complex. Not only are patients concerned with the malalignment of teeth, they have expectations related to tooth size, color, and shape. Some patients have a stronger focus on esthetic issues than on issues associated with function. In other research, preferences of dentists, dental students, and men and women patients have been shown to differ significantly for pro- 
portion and symmetry of teeth or fabricated restorations.

One important and consistent finding has emerged from studies done on professional, peer, and self-perceptions of facial attractiveness, and that is that an individual's desire for treatment cannot be equated with his or her clinical need for oral health-related treatment. The point emphasized is that treatment is not only restricted to restoration of form and function. By the end of the restoration process, after many discussions with the clinician, the patient's reasoning for treatment may include improved function or comfort, but it is likely that the focus at the start may have been mainly for esthetic improvement. Professional perceptions and peer perceptions of appearance can be part of a social-clinical dynamic that the individual uses to develop and maintain his or her self-perception.

More specifically, self-perceptions of attractiveness are founded in individual experiences, through face-to-face interactions. Clinicians' perceptions are shaped by a different set of experiences; experiences based on familiarity with a much broader range of defects and greater variability with respect to esthetic impact. Clinical perceptions are interpreted against treatment possibilities and an understanding of the limitations of techniques and results. Yet, in the clinical setting and during the decision-making that occurs there, these different perspectives and the concerns that underlie them will influence one another, both directly and indirectly. There is considerable anecdotal evidence that surgeons and other practitioners listen carefully to their patients' concerns about appearance, yet patients' perceptions and expectations are shaped by their care provider's views.

Reprise. The influence of social and economic factors in prosthodontics can be derived from social, psychologic, and economic facts. As the population ages there will be an increased need for the rerestoration of teeth with an increasing emphasis on fixed and removable partial prosthodontics. There will be increasing discrepancies in individual ability to pay for invasive treatment. Responses to aging, physical decline, and facial attractiveness are highly individual and personal and may not reflect the clinicians' evaluations for severity of esthetic impairment. Indeed, patient desire for esthetic improvement may be in direct conflict with the clinical judgment or treatment philosophy of the dentist when form and function are not in need of restoration.

\section{IMPLICATION FOR PATIENT CARE: THE BIOPSYCHOSOCIAL MODEL}

For far too long, the traditional practice of medicine and dentistry has separated the body from the mind. (Dentistry is even described as separating the mouth from the body.) We deny the humanity behind the clinical problem if we evaluate a condition, diagnose a problem, or design a treatment without full consideration of the patient's thoughts, expectation, desires, reservations, or lifestyle.

We invoke the biopsychosocial model for care, the patient-centered model for care, instead of the more expedient, clinician-centered biomedical model if we seriously and consistently question and listen to the patient as part of all phases of treatment. Health in the biopsychosocial model is influenced or defined by biologic factors, but also by psychologic factors, for example, thoughts and feelings, and social factors, such as the influence of family, friends, and the environment. ${ }^{19,20}$ The biopsychosocial model, from health psychology and behavioral medicine, is a branch of applied psychology that uses theory and data to help solve problems related to improving health, promoting wellness, and preventing disease. This model places an emphasis on multidisciplinary team approaches to diagnosis and treatment planning, targets therapy uniquely to an individual, and makes explicit the relationship between the patient and practitioner. ${ }^{20}$

Treatment outcomes across the full scope of prosthodontics can be improved by the use of a biopsychosocial approach. Can we, with equal validity and reliability, develop systems to evaluate patients' thoughts and feelings related to treatment or identify the social factors that will enhance or detract from treatment? Can we reformulate our thinking about the length of prosthodontic treatment to include long-term follow-up to preserve the restored dentition? How should we proceed if we have reason to believe that a patient will have difficulty maintaining optimal oral hygiene required to preserve the restorations? Are we able to predict accurately which patients will assume personal responsibility and commitment to homecare after experiencing the process of restoration, improved function, increased comfort, and enhanced esthetics? The "intervention" of treatment may well provide the needed experience on the part of the patient to alter beliefs, attitudes, values, and behavior related to oral health. Longterm follow-up should include evaluation, feedback, and discussion of how successful the patient has been in the maintenance of the restorations, to prevent future decline or relapse. ${ }^{21}$

\section{IMPLICATIONS FOR EDUCATION}

The process of education for clinicians is as central to patient care as the actual provision of treatment itself. Teaching beyond technique orthodoxy is an acknowledged challenge where treatment is invasive, irreversible, surgical, and rehabilitative. What do patients need to be better served and what are the implications for educational programs? Should explicit assessments of social, psychological, and economic influences be included in prosthodontic treatment? Will patients be better served by such assessments? Would providers of care benefit? Would learning the art and science of dentistry be improved? The importance of lifestyle, behavior, and social and economic environments are inextricably tied to demography and changing treatment needs. The guidance provided by acknowledging these influences eliminates the risk of placing the patient outside the center of the treatment process. 


\section{Education guidelines}

How do we keep the patient at the center of treatment? Psychology and behavioral sciences are both listed as recommended and necessary topics in the prosthodontics education guidelines from 1969 and $1983 .{ }^{22,23}$ Both documents included material from the "behavioral world." However, the discipline is not in balance as a biologic, rehabilitative, and psychosocial entity. The lack of balance is everywhere in medical and dental education at both the predoctoral and graduate levels. Only over the past 5 years has the lack of balance and its implications for treatment, prevention of disease, and promotion of health been addressed.

When the curriculum and educational challenges for presenting a balance of material to best serve students and patients are considered, there is less concern with providing enough information in one area or another. Instead, concern grows increasingly with how we are teaching our students, predoctoral and postgraduate, how to make sense of the information we are asking them to master. Landesman's 1976 prediction ${ }^{24}$ indicated that population treatment needs would produce a concomitant pedagogic need for prosthodontic educators "who have the ability to guide students in securing the amount and quality of experience that will promote the optimum development of their potential." Landesman's prescience further suggested that the chief characteristic of the "teachers of tomorrow" would be their ability to promote an "ongoing exchange of ideas." Embedded in this phrase is the notion that we must see all sides of treatment solutions, and this includes the patient's side. The implications of "seeing all sides" are profound, especially when the psychological, social, and economic sides of treatment decisions are considered. Two patients can have nearly the same anatomic and biologic status, defining treatment solutions that are practically similar. However, when all the other nonanatomic, nonbiologic aspects of treatment are considered, two entirely different solutions may be required. How do we teach students to think about such solutions? How do we present solutions free of personal preferences, attitudes, and orthodoxy? This challenge, predicted in 1976, is alive and with us today.

\section{Education for judgment: Developing a new breed of practitioner}

The educational literature from the past 5 to 8 years has been filled with research evidence and impassioned debate $^{25}$ on the virtues of active learning over teachercentered learning. Teacher-centered learning is the familiar lecture and slides approach. To this day, it is the method that predominates the predoctoral curriculum. Active learning, where questions and answers from students and teachers are equally important to the learning process, is the predominant activity of postgraduate education. Many classes of postgraduate students have learned how to think creatively about dentistry only when they studied their specialty. This situation is a function of time, experience, maturation, and development of skill. The lack of progress in predoctoral pedagogy in particular only delays the mastery of knowledge, the retention of information, and the development of wisdom-characteristics of thinking that will ultimately best serve the needs of our patients. Skill mastery should be balanced with questioning in educational approaches to provide the highest quality of care to patients.

There is a real need for the knowledge, information, and wisdom of the specialist to help develop new specialists, but also solid thinking general dentists. Finding the resources, time, faculty, and funding to plan and implement new ways of teaching in dentistry will be almost as difficult as finding the will.

\section{CONCLUSION}

Medical and dental education is undergoing reform all over the world, focused on both content and pedagogy, what we teach and how we teach. ${ }^{26-28}$ Some of this reform includes new attitudes and values for the clinician-patient relationship in dentistry; and this information is maximally useful when placed in the context of understanding patient needs from the patient's perspective.

Brewer $^{7}$ said the following about dental education and practice, which embraces the biopsychosocial model:

\begin{abstract}
When I was in dental school, we didn't get any credit for talking to or listening to the patient. We only got credit for doing something technical to the patient. So many dentists figure that they're not doing anything unless they are making something for the patient. We as dentists don't want to impose our esthetic values upon the patient. Give the patient ample time to examine the [restorations] ... Because the patient cannot see himself [herself] as others will see him [her], it is important to seek the participation of the patient's family member or friends at this time ... [T] his is a most appropriate time to talk and especially listen to the patient [p. 142].
\end{abstract}

Leadership and willingness to work in new and different ways to define standards and design curriculum for patient-centered care will be needed as models for students to observe and imitate. Students will need to gain knowledge and skill and learn positive attitudes towards patients. They will need to learn how to feel and talk about their concerns during the course of treatment and manage the discussion of patients' concerns. Recent descriptions of prosthodontic education in four world regions did not include a noticeable dedication of curriculum for training in patient-centered or biopsychosocial approaches. ${ }^{29-32}$ Programs based on the patient-centered philosophy will take thoughtful reorientation in didactic and clinical teaching environments.

\section{REFERENCES}

1. British Society for the Study of Prosthetic Dentistry. Guides to standards in prosthetic dentistry. Br Dent J 1981;150:167-9. 
2. Academy of Prosthodontics. Principles, concepts, and practices in prosthodontics. J PROSTHET DENT 1989;61:88-109.

3. Council on Dental Materials, Instruments, and Equipment. American National Standards/American Dental Association Specifications. J Am Dent Assoc 1983;107:640-1.

4. Melamed BG. Psychological considerations for implant patients. J Oral Implantol 1989;15:249-54.

5. Kent G, Johns R. A controlled longitudinal study on the psychologicalal effects of osseointegrated dental implants. Int $J$ Oral Maxillofac Implant 1991;6:470-4.

6. Harle TJ, Anderson JD. Patient satisfaction with implant-supported prostheses. Int J Prosthodont 1993;6:153-62.

7. Halperin AR, Graser GN, Rogoff GS, Plekavich EJ. Mastering the art of complete dentures. Chicago: Quintessence Publ, 1988.

8. Bolender CL, Swoope CC, Smith DE. The Cornell Medical Index as a prognostic aid for complete dentures. J PROSTHET DENT 1969;22:20-9.

9. Kiyak HA, Beach BH, Worthington P, Taylor T, Bolender C, Evans J. The psychological impact of osseointegrated dental implants. Int J Oral Maxillofac Implant 1990;5:61-9.

10. van Waas MA. The influence of psychological factors on patient satisfaction with complete dentures. J PROSTHET DeNT 1990;63:545-8.

11. van Waas MA. Determinants of dissatisfaction with dentures: a multiple regression analysis. J Prosthit DeNT 1990;64:569-72.

12. Longino CF Jr, Crown WH. Older Americans: rich or poor. Am Demograph 1991;13:48-54.

13. Waldrop J. Secrets of the age pyramids. Am Demograph 1992;14:46-53.

14. White RP, Formicola AJ. Dentistry a profession in transition. In: Simonsen RJ, ed. Dentistry in the 21st century a global perspective. Chicago: Quintessence Publ Co, 1991:173-90.

15. Douglass CW, Furino A. Balancing dental service requirements and supplies: epidemiologic and demographic evidence. J Am Dent Assoc 1990;121:587-92.

16. Braun F. Dentistry in the 21st century, practice: Germany. In: Simonsen RJ, ed. Dentistry in the 21st century a global perspective. Chicago: Quintessence Publ Co, 1991:221-34.

17. Kerr P. Shift for marketers: yup to grump. New York Times, August 27, 1991:D1-6.

18. Albino JE, Alley TR, Tedesco LA, Tobiasen JA, Kiyak HA, Lawrence SD. Esthetic issues in behavioral dentistry. Ann Behav Med 1990;12:14855 .
19. Friedman HS, DiMatteo MR. Health psychology. Englewood Cliffs, NJ: Prentice Hall, 1989.

20. Taylor SE. Health psychology. New York: Random House Co, 1986.

21. Tedesco LA, Keffer MA, Davis EL. Social cognitive theory and relapse prevention: reframing patient compliance. J Dent Educ 1991;55:575-81.

22. Boucher LJ. Guidelines for advanced prosthodontic education-1969. J ProstheT DeNT 1970;23:104-10.

23. Secretary, Commission on Dental Accreditation. Requirements for advanced specialty education programs in prosthodontics. J ProstHET DENT 1983;50:836-43.

24. Landesman HM. The making of a prosthodontist: advanced prosthodontic education at the University of Southern Californiu. J Prosther I DENT 1976;35:580-4.

25. Christensen CR, Garvin DA, Sweet A. Education for judgment: the artistry of discussion leadership. Boston: Harvard Business School Press, 1991

26. Tedesco LA. Responding to educational challenges with problem-based learning and information technology. J Dent Educ 1990;54:544-7.

27. Project Panel on the General Professional Education of the Physician and College Preparation for Medicine (Association of American Medical Colleges). J Med Educ 1984;59:11.

28. Shugars DA, O'Neil EH, Bader JD, Pew Health Profession Commission. Healthy America: practitioners for 2005, an agenda for action for U.S. health professional schools. Durham, NC: The Pew Health Professions Commission, 1991.

29. DeBoever JA. Undergraduate and graduate education in prosthodontics in continental Europe. Int J Prosthodont 1989;2:28-34.

30. Tsuru H. Prosthodontics education in Japan. Int $J$ Prosthodont 1989;2:35-41.

31. MacGregor AR. Education in prosthodontics. Britain, South Africa, and Australia. Int J Prosthodont 1989;2:185-9.

32. Preston JD. Prosthodontic education in the United States. Int J Prosthodont 1989;2:190-5.

Reprint requests to:

DR. LISA A. Tedesco

Office of Academic Affairs

SCHOOL OF DENTISTRY

UNIVERSTTY OF MICHIGAN

ANN ARBoR, MI 48109-1078 